\title{
Vacancy Formation and Oxidation Characteristics of Single Layer $\mathrm{TiS}_{3}$
}

\author{
F. Iyikanat, ${ }^{* \dagger}$ H. Sahin, ${ }^{*} \ddagger$ R. T. Senger, ${ }^{\dagger}$ and F. M. Peeters ${ }^{\ddagger}$ \\ ${ }^{\dagger}$ Department of Physics, Izmir Institute of Technology, 35430 Izmir, Turkey \\ ${ }^{\ddagger}$ Department of Physics, University of Antwerp, Groenenborgerlaan 171, B-2020 Antwerpen, Belgium
}

ABSTRACT: The structural, electronic, and magnetic properties of pristine, defective, and oxidized monolayer $\mathrm{TiS}_{3}$ are investigated using first-principles calculations in the framework of density functional theory. We found that a single layer of $\mathrm{TiS}_{3}$ is a direct band gap semiconductor, and the bonding nature of the crystal is fundamentally different from other transition metal chalcogenides. The negatively charged surfaces of single layer $\mathrm{TiS}_{3}$ makes this crystal a promising material for lubrication applications. The formation energies of possible vacancies, i.e. $\mathrm{S}, \mathrm{Ti}, \mathrm{TiS}$, and double $\mathrm{S}$, are investigated via total energy optimization calculations. We found that the formation of a single $S$ vacancy was the most likely one among the considered vacancy types. While a single $S$ vacancy results in a nonmagnetic, semiconducting character with an enhanced band gap, other vacancy types induce metallic behavior with spin polarization of $0.3-0.8 \mu_{\mathrm{B}}$. The reactivity of
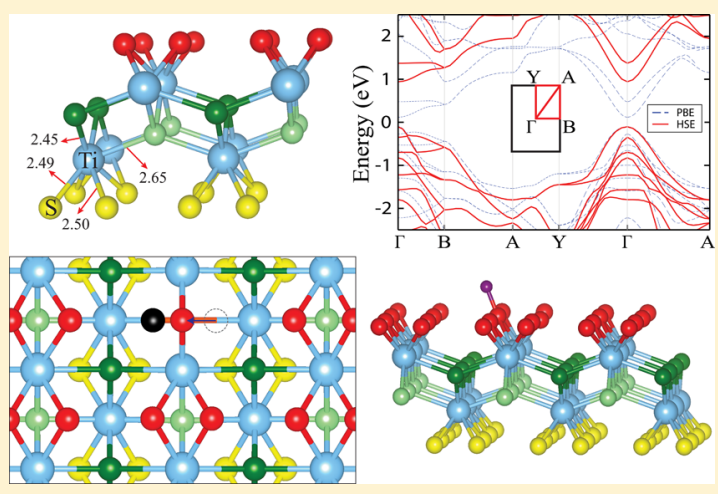
pristine and defective $\mathrm{TiS}_{3}$ crystals against oxidation was investigated using conjugate gradient calculations where we considered the interaction with atomic $\mathrm{O}, \mathrm{O}_{2}$, and $\mathrm{O}_{3}$. While $\mathrm{O}_{2}$ has the lowest binding energy with $0.05-0.07 \mathrm{eV}, \mathrm{O}_{3}$ forms strong bonds stable even at moderate temperatures. The strong interaction $\left(3.9-4.0 \mathrm{eV}\right.$ ) between atomic $\mathrm{O}$ and $\mathrm{TiS}_{3}$ results in dissociative adsorption of some O-containing molecules. In addition, the presence of S-vacancies enhances the reactivity of the surface with atomic $\mathrm{O}$, whereas it had a negative effect on the reactivity with $\mathrm{O}_{2}$ and $\mathrm{O}_{3}$ molecules.

\section{INTRODUCTION}

The wide range of different structural forms and the tunability of their electronic properties have made two-dimensional ultrathin materials popular in the field of condensed matter physics. In the past decade, following the synthesis of graphene, ${ }^{1,2}$ layered crystals of transition metal dichalcogenides (TMDs) have attracted tremendous interest owing to their remarkable electronic, mechanical, and optical properties. ${ }^{3-5}$ Depending on the combination of metal and chalcogen atoms, TMDs may display metallic, semimetallic, semiconducting, and even superconducting behavior. ${ }^{6-11}$ Various experimental methods to thin down TMDs to monolayers ${ }^{12-14}$ and a number of theoretical studies revealing the possibility of tuning their properties have already been reported. ${ }^{15-18}$ In addition to TMDs $\left(\mathrm{MoX}_{2}, \mathrm{X}=\mathrm{S}\right.$, Se, Te $)$ having graphene-like crystal structure, recent efforts have also led to the synthesis of novel TMDs with entirely different crystal structures. Titanium trisulfide $\left(\mathrm{TiS}_{3}\right)$ is one of the most recent examples of such layered transition metal chalcogenides.

The atomic structure belongs to the space group $P 2_{1} / m$, and $\mathrm{TiS}_{3}$ crystallizes in bundles of molecular chains that are formed by trigonal prisms, where the metal atoms occupy the centers of the prisms. ${ }^{19,20}$ Finkman et al. ${ }^{21}$ have shown that $\mathrm{TiS}_{3}$ crystals are semiconducting with extrinsic n-type conductivity that have room temperature mobility of $30 \mathrm{~cm}^{2} /(\mathrm{V} \mathrm{s})$. Recently, strong nonlinearity of the current-voltage characteristics has been reported by Gorlava et al. ${ }^{22,23}$ In contrast to many other layered TMDs which exhibit an indirect to direct band gap transition at the monolayer limit, $\mathrm{TiS}_{3}$ exhibits a direct band gap even for a width of hundreds of layers. Direct optical transitions with a band gap of $1.10 \mathrm{eV}$ have been detected in thin films of $\mathrm{TiS}_{3}{ }^{24}$ Moreover, these thin films show photocurrent response to white light illumination. ${ }^{25}$

Using high-resolution TEM for defective crystals, a metal-toinsulator transition with charge localization below $T_{\mathrm{MI}} \approx 325 \mathrm{~K}$ has been proposed by Guilmeau et al. ${ }^{26}$ Their studies revealed that $\mathrm{TiS}_{3}$ has low thermal conductivity and a large absolute value of the Seebeck coefficient at high temperatures. In a recent study by Island et al. ${ }^{27}$ field effect transistors (NR-FET) have been fabricated at room temperature by isolating few-layer $\mathrm{TiS}_{3}$ nanoribbons. The electron mobility of few-layer $\mathrm{TiS}_{3}$ was found to be $2.6 \mathrm{~cm}^{2} /(\mathrm{V} \mathrm{s})$ and exhibits n-type semiconductor behavior with ultrahigh photoresponse and fast switching times. Furthermore, Island et al. ${ }^{28}$ have isolated single-layer $\mathrm{TiS}_{3}$ by mechanical exfoliation.

In this study, motivated by the latest experimental ${ }^{27-32}$ and theoretical ${ }^{33,34}$ studies on $\mathrm{TiS}_{3}$, we investigated (i) structural and electronic properties of single layer $\mathrm{TiS}_{3}$, (ii) formation of vacancies in the pristine material and their influence on electronic properties, and (iii) environmental stability of its surface against oxidation. The paper is organized as follows. In section II, we present the details of the computational

Received: February 15, 2015

Revised: April 23, 2015

Published: April 28, 2015 
methodology. Geometric, electronic, and magnetic properties of defect-free $\mathrm{TiS}_{3}$ and of various vacancy defects are given in sections III and IV, respectively. The response of pristine and defective $\mathrm{TiS}_{3}$ to oxidation is analyzed in section V. Results are discussed in section VI.

\section{COMPUTATIONAL METHODOLOGY}

All the calculations were performed within the spin-polarized density functional theory (DFT) using projector-augmentedwave potentials (PAW) and a plane-wave basis set as implemented in the Vienna $a b$ initio simulation package (VASP). ${ }^{35,36}$ The cutoff energy for the plane waves was chosen to be $500 \mathrm{eV}$. The Perdew-Burke-Ernzerhofs (PBE) version of generalized gradient approximation $(\mathrm{GGA})^{37}$ was used for the description of the exchange correlation functional. For a better approximation of band gap values, underestimated by PBE functional, the HSE06 functional was also used. ${ }^{38}$ In the HSE06 approach, the fraction of the Hartree-Fock exchange and the screening parameter were set to $\alpha=0.25$ and $0.2 \AA^{-1}$, respectively.

Spin-polarized calculations were performed in all cases, and atomic charges were calculated using the Bader charge population analysis. ${ }^{39}$ The Gaussian smearing method was employed for total energy calculations with a width of $0.01 \mathrm{eV}$. Density functional theory plus the long-range dispersion correction (DFT + D2) method was used to calculate the nonlocal correlation energies. ${ }^{40} C_{6}$ values of $\mathrm{Ti}$ and $\mathrm{S}$ atoms were 10.800 and 5.570, respectively. 1.562 and 1.683 were used as the vdW radius of $\mathrm{Ti}$ and $\mathrm{S}$ atoms, respectively. ${ }^{40}$

Geometric structures of the perfect, vacancy defected, and oxidized monolayer $\mathrm{TiS}_{3}$ were fully optimized to minimize each component of the interatomic Hellmann-Feynman forces until a precision of $10^{-4} \mathrm{eV} / \AA \AA$ was reached. The pressure in the unit cell was kept below 1 kbar. The convergence criterion for energy was chosen to be $10^{-5} \mathrm{eV}$ between two consecutive steps. The conjugate gradient method was used to compute lattice constants and total energies. In all cases, lattice parameters were optimized along the $a_{1}$ and $a_{2}$ directions. In order to hinder interlayer interaction within the periodic images, a vacuum spacing of $16 \AA$ between adjacent layers was chosen. The lateral distance between vacancies (adsorbed atoms) was at least $10 \AA$ in order to eliminate the interaction between vacancies (adsorbed atoms) in neighboring supercells. For the unit cell of $\mathrm{TiS}_{3}, 19 \times 19 \times 1$ k-point mesh was used in PBE calculations, whereas due to high computational cost, the k-point mesh was reduced to $5 \times 5 \times 1$ in HSE calculations. For the supercell calculations, the same k-point mesh of $3 \times 3$ $\times 1$ was used for both PBE and HSE. The vacancies were obtained by removing the considered atoms from a $3 \times 3$ supercell which consist of $18 \mathrm{Ti}$ atoms and $54 \mathrm{~S}$ atoms.

The calculated formation energies of the vacancies was obtained from $E_{\mathrm{F}}=E_{\mathrm{M}-\mathrm{A}}-E_{\mathrm{M}}+n E_{\mathrm{A}}$, where $E_{\mathrm{F}}$ is the formation energy of the relevant vacancy, $E_{\mathrm{M}-\mathrm{A}}$ is the total energy of the supercell with vacancy, $n$ is the number of removed atoms, $E_{\mathrm{A}}$ is the energy of the removed atom, and $E_{\mathrm{M}}$ is the energy of monolayer $\mathrm{TiS}_{3}$. Binding energies of $\mathrm{O}, \mathrm{O}_{2}$, and $\mathrm{O}_{3}$ were calculated for the most favorable adsorption sites. These binding energies were calculated from the expression $E_{\mathrm{B}}$ $=E_{\mathrm{M}+\mathrm{A}}\left(E_{\mathrm{D}+\mathrm{A}}\right)-E_{\mathrm{M}}\left(E_{\mathrm{D}}\right)-m E_{\mathrm{A}}$, where $E_{\mathrm{B}}$ is the binding energy of the adsorbed atom on pristine ( $S$ defected) $\mathrm{TiS}_{3}, m$ is the number of adsorbed atoms, $E_{\mathrm{M}}\left(E_{\mathrm{D}}\right)$ and $E_{\mathrm{M}+\mathrm{A}}\left(E_{\mathrm{D}+\mathrm{A}}\right)$ are the total energy of pristine ( $S$ defected) and the adsorbed atompristine (adsorbed atom-S-defected) system, respectively.

\section{PRISTINE SINGLE LAYER TiS}

The unit cell of monolayer $\mathrm{TiS}_{3}$ is a rectangular prism and is composed of two $\mathrm{Ti}$ atoms and six $\mathrm{S}$ atoms. The coordination of these atoms is illustrated by the top and tilted-side views in Figures $1 \mathrm{a}$ and $1 \mathrm{~b}$, respectively. The monolayer $\mathrm{TiS}_{3}$ is
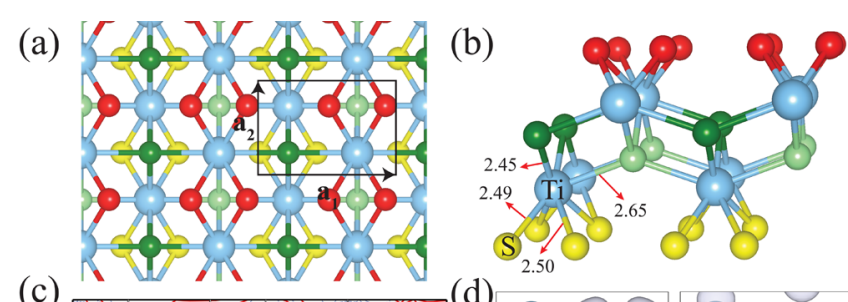

(c)

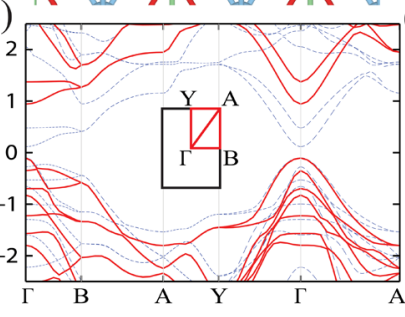

(d)

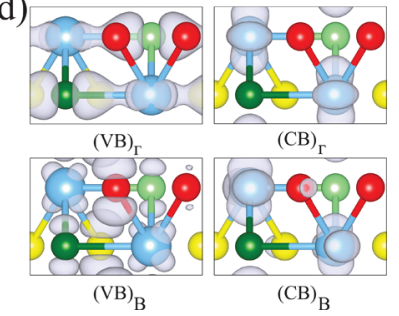

Figure 1. (a) Top view of monolayer $\mathrm{TiS}_{3}$ and its unit cell shown by a rectangle. For a better top view, $\mathrm{S}$ atoms in different layers are presented by different colors. (b) Tilted side view of $2 \times 2 \times 1$ supercell of monolayer $\mathrm{TiS}_{3}$. (c) Blue dashed lines and red lines illustrate PBE and HSE06 results for the electronic band diagram of monolayer $\mathrm{TiS}_{3}$, respectively. (d) Band decomposed charge densities of valence band (VB) and conductance band (CB) at $\Gamma$ and $\mathrm{B}$.

composed of chain-like structures consisting of trigonal prisms with the metal atom occupying the centers of these prisms. In this phase, chains are parallel to the $a_{2}$, and they form layers in the $a_{1} a_{2}$ plane, which are coupled with each other by the van der Waals interaction to form the bulk layered structure of $\mathrm{TiS}_{3}$. Our calculations show that monolayer $\mathrm{TiS}_{3}$ has lattice vectors $a_{1}=4.99 \AA$ and $a_{2}=3.39 \AA$. These lattice parameters are comparable to the experimental bulk results $a_{1}=4.958 \AA$, $a_{2}=$ $3.4006 \AA, a_{3}=8.778 \AA$, and $\beta=97.32 .^{19,20}$ As shown in Figure $1 \mathrm{~b}$, the bond distances of $\mathrm{Ti}$ atom and two different surface $S$ atoms (red or yellow $S$ atoms) are 2.49 and $2.50 \AA$, respectively. At the same time, these two surface $S$ atoms are two of the three base $S$ atoms of the trigonal prisms. The bond distance of $\mathrm{Ti}$ atom and third base $\mathrm{S}$ atom (dark or light green $\mathrm{S}$ atoms) of the trigonal prism is $2.45 \AA$. The atom-atom distance of $\mathrm{Ti}$ atom and $S$ atom (light or dark green $S$ atoms) of neighboring prism is $2.65 \AA$.

Charge density analysis is an efficient way to discuss the character of the interatomic interactions and bonding. In Figure 2 , we present a cross section of the $3 \mathrm{D}$ total charge density. Bader charge analysis shows that in parallel to their electronegativity values a significant amount of charge is transferred from $\mathrm{Ti}$ to the $\mathrm{S}$ atoms. While two $\mathrm{S}$ atoms at the surface (shown by red balls) share 0.7 electrons donated by the underlying $\mathrm{Ti}$ atom, 0.8 electron transfer occurs from $\mathrm{Ti}$ to $\mathrm{S}$ atom in the middle of the crystal (shown by light green balls). Thus, Ti-S bonds between surface and inner $\mathrm{S}$ atoms have an entirely different character. As is also delineated in Figure 2, the bond between $\mathrm{Ti}$ and inner $\mathrm{S}$ atoms is constructed through 0.8 electron transfer, and therefore it has an ionic character. However, the bonds between $\mathrm{Ti}$ and surface $\mathrm{S}$ atoms are constructed through relatively less electron transfer, and hence it has mostly covalent character. It is also seen from Figure 2 


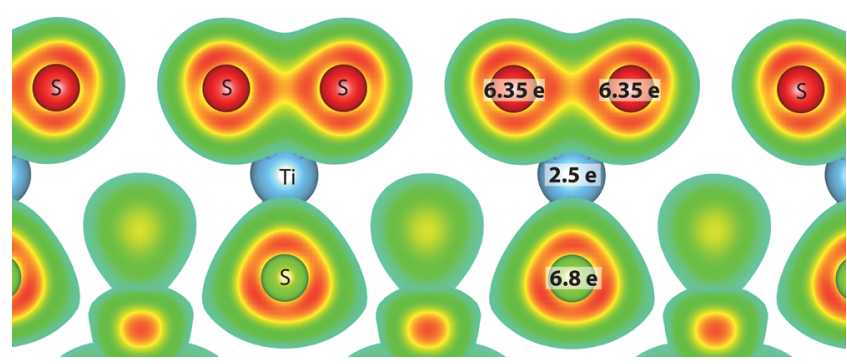

Figure 2. Cross-sectional plane view of the charge densities, with two surface, and one inner $S$ atom placed at this plane. Valence charges on inner and outer $\mathrm{S}$ atoms are also shown. Color code of atoms is the same as in Figure 1. Electron density increases from green to red.

that each surface atom interacts with neighboring $S$ atoms while there is no contact between the inner $S$ atoms. Therefore, one expects anisotropic electronic and transport properties due to the varying character of the surface states along $a_{1}$ and $a_{2}$ directions. It is also worth mentioning that the negatively charged surface of monolayer $\mathrm{TiS}_{3}$ may find interesting applications such as nanoscale lubricants and charged coatings.

Calculated band diagrams of pristine $\mathrm{TiS}_{3}$ using $\mathrm{PBE}$ and HSE06 methods are shown in Figure 1c. It is known that often the values, obtained with the PBE functional, underestimate the energy band gaps of semiconductors. The band gap of $\mathrm{TiS}_{3}$ is calculated to be $0.23 \mathrm{eV}$ by using the PBE approximation. However, including the HSE06 correction, one gets a $1.05 \mathrm{eV}$ direct band gap at the $\Gamma$ point, which is in good agreement with the experimental value of $1.10 \mathrm{eV}$ for few-layer $\mathrm{TiS}_{3}{ }^{24,25}$ For further analysis of the band structure of $\mathrm{TiS}_{3}$, charge densities of the valence band maximum (VB) and the conduction band minimum (CB) at the $\Gamma$ and the $\mathrm{B}$ high-symmetry points are shown in Figure 1d. It is seen that the VB is composed of $2 \mathrm{p}_{x}$ orbitals of $\mathrm{S}$ and $3 \mathrm{~d}_{x z}$ orbitals of $\mathrm{Ti}$, and they form a strong bond, whereas the main contribution to the $\mathrm{CB}$ edge comes from $3 \mathrm{~d}_{y^{2}-z^{2}}$ orbitals of $\mathrm{Ti}$ atom at the $\Gamma$ point. At the $\mathrm{B}$ point, $\mathrm{p}_{y}$ orbitals of $\mathrm{S}$ atoms dominant while $3 \mathrm{~d}_{x y}$ orbitals of $\mathrm{Ti}$ atom contribute slightly to the VB. In particular, $\mathrm{p}$ orbitals of surface $S$ atoms are larger than $\mathrm{p}$ orbitals of the inner $\mathrm{S}$ atoms. Thus, the main contribution to the VB is dominated by $\mathrm{p}_{y}$ orbitals of the surface $S$ atoms. Like at the $\Gamma$ point, while the $S$ atoms do not contribute significantly, all the contribution comes from the $3 \mathrm{~d}_{y^{2}-z^{2}}$ orbitals of the Ti atom to the CB. In the trigonal prisms every $\mathrm{Ti}$ atom shares 4 valence electrons with 3 base $S$ atoms and 1 neighboring chain $S$ atom. Because of the lack of any unsaturated orbitals, monolayer $\mathrm{TiS}_{3}$ has a nonmagnetic ground state.

\section{DEFECTIVE SINGLE LAYER TiS}

A. S Vacancy. The first defective structure we consider is a single $\mathrm{S}$ vacancy in $\mathrm{TiS}_{3}$ monolayer. To compare the geometric structure of monolayer $\mathrm{TiS}_{3}$ in the presence and absence of a vacancy, a $3 \times 3$ supercell is considered, and the lattice vectors for the defect-free $\mathrm{TiS}_{3}$ computational supercell are found as $a_{1}$ $=14.98 \AA$ and $a_{2}=10.18 \AA$. Fully relaxed geometric structure when a single $S$ atom is removed from the surface of monolayer $\mathrm{TiS}_{3}$ is shown in Figure 3a. When $S$ vacancy is introduced, the lattice vectors of $3 \times 3$ supercell change and become $a_{1}=14.90$ $\AA$ and $a_{2}=10.17 \AA$. Thus, the presence of the $S$ vacancy leads to a minute shrinkage of the lattice vectors of $\mathrm{TiS}_{3}$. As can be seen from Figure 3a, the nearest surface $S$ atom to the vacancy follows the direction of the arrow, localizes on top of the inner
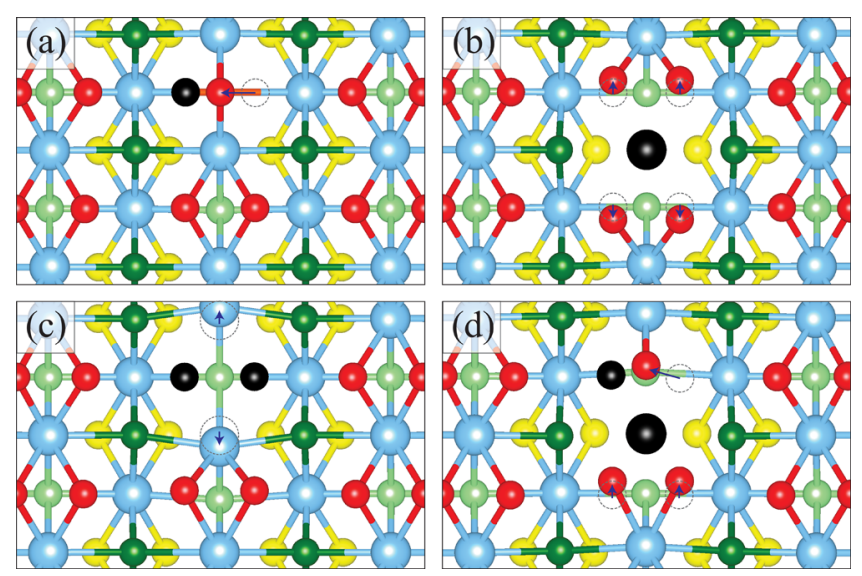

Figure 3. Top view of relaxed monolayer $\mathrm{TiS}_{3}$ with (a) $\mathrm{S}$ vacancy, (b) Ti vacancy, (c) double $S$ vacancy, and (d) TiS vacancy. Black atoms illustrate removed atoms, and dashed circles show initial position of the displaced atom. Color code of the other atoms is the same as in Figure 1. The directions of displaced atoms are depicted by blue arrows.

$S$ atom (light green) and forms reconstructed bonds with the nearest $\mathrm{Ti}$ atoms with a bond length of $2.32 \AA$. Bader charge analysis tells us that the total charge of this atom is increased by 0.4 electron when removing the nearest-neighbor $S$ atom. The charges of other atoms do not change significantly with the removal of the surface $S$ atom. Our calculated results show that the formation energy of an $\mathrm{S}$ vacancy is $3.58 \mathrm{eV}$.

To calculate the electronic properties of $S$ vacancy defected monolayer $\mathrm{TiS}_{3}$ in addition to PBE, the HSE06 method is also used, and the results are listed in Table 1. Moreover, the density of states (DOS) of pristine and $\mathrm{S}$ atom-removed $\mathrm{TiS}_{3}$ calculated by PBE method are shown in Figures $4 \mathrm{a}$ and $4 \mathrm{~b}$, respectively. As can be seen from the figures and Table 1, removing one $\mathrm{S}$ atom from the surface of $\mathrm{TiS}_{3}$ does not make any notable effect on the electronic structure of $\mathrm{TiS}_{3}$. The monolayer conserves its semiconductor character. Because of the reconstruction of the $S$ atom and its binding with $2 \mathrm{Ti}$ atoms, there are no unsaturated bonds. Thus, the nonmagnetic character of the $\mathrm{TiS}_{3}$ is preserved during the formation of an $\mathrm{S}$ vacancy.

B. Ti Vacancy. Relaxed geometric structure when a single $\mathrm{Ti}$ atom is extracted from $\mathrm{TiS}_{3}$ is illustrated in Figure 3b. A significant surface reconstruction is observed after geometric relaxation. This figure shows that, when one $\mathrm{Ti}$ atom is taken out, the displacement of the inner $\mathrm{S}$ atoms that were attached to it is not significant; nevertheless, they lose 0.1 electrons. However, the four surface $S$ atoms are released, they move toward the other $\mathrm{Ti}$ atoms, and they lose approximately 0.2 electrons. This gives rise to an expansion in the lattice parameter $a_{1}$, which after relaxation becomes $a_{1}=15.05 \AA$ and $a_{2}=10.14 \AA$. Hence, removal of Ti atom leads to an increase in $a_{1}$, whereas a decrease in $a_{2}$. The formation energy of $\mathrm{Ti}$ vacancy is $12.00 \mathrm{eV}$. Defects with high formation energies are unlikely to form, and therefore an $S$ vacancy will have a much higher probability to form as compared to a Ti vacancy.

Unlike the $\mathrm{S}$ vacancy, the Ti vacancy has a major effect on the electronic structure of $\mathrm{TiS}_{3}$; it loses its semiconductor character and becomes metallic. As shown in Figure 4c, the states orginating from $\mathrm{Ti}$ vacancy are near the VB with three peaks of gap states arising from the mixture of the orbitals of the neighboring $\mathrm{S}$ and $\mathrm{Ti}$ atoms. This leads to the possibility of 
Table 1. Lattice Parameters, Formation Energies, Magnetic Moments, Electronic Characteristics, and Band Gaps of $3 \times 3$ Supercell of Monolayer $\mathrm{TiS}_{3}$ and Its Few Defected Forms Calculated Using the PBE Method as Well as HSE06 Results for the Band Gap of Semiconductors

\begin{tabular}{lcccc} 
& $a_{1} / a_{2}(\AA)$ & $E_{\mathrm{F}}(\mathrm{eV})$ & $m(\mu \mathrm{B})$ & electronic characteristic \\
pristine $\mathrm{TiS}_{3}$ & $14.98 / 10.18$ & & 0.0 & semiconductor \\
$\mathrm{S}$ vacancy & $14.90 / 10.17$ & 3.58 & 0.0 & semiconductor \\
Ti vacancy & $15.05 / 10.14$ & 12.00 & 0.5 & metal \\
double S vacancy & $14.96 / 10.18$ & 8.48 & 0.8 & metal \\
Ti, S vacancy & $14.90 / 10.13$ & 16.15 & 0.3 & metal \\
\hline
\end{tabular}

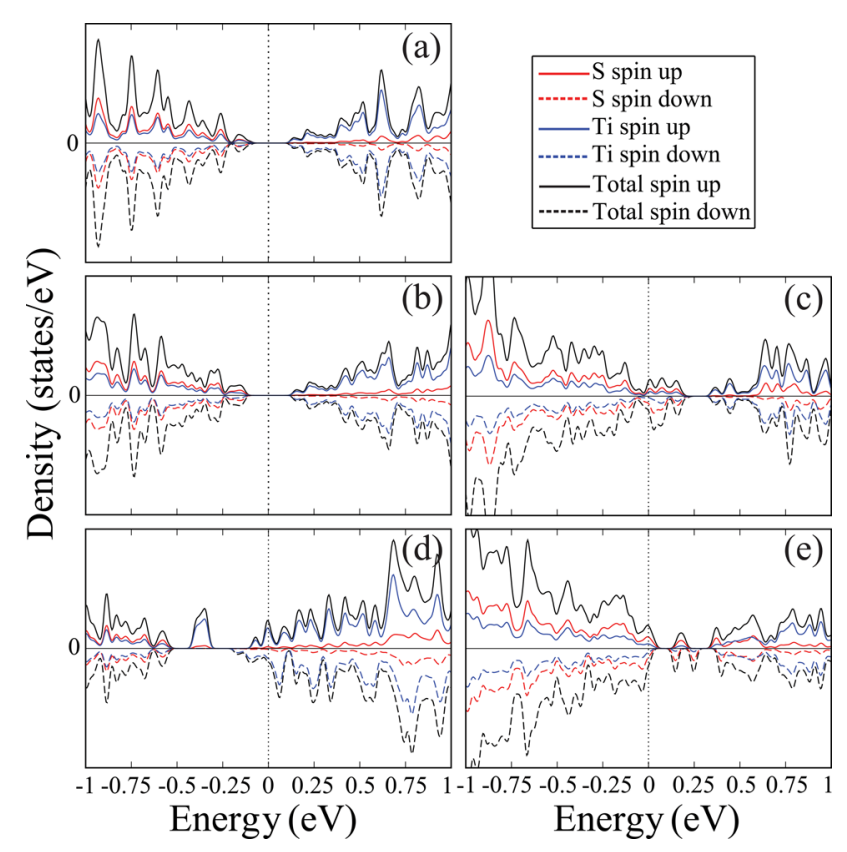

Figure 4. Density of states of (a) pristine $\mathrm{TiS}_{3}$, with (b) S vacancy, (c) Ti vacancy, (d) double S vacancy, and (e) TiS vacancy.

p-type doping if Ti vacancy can be created. As seen from Figure $4 c$, at the Fermi level, there is a slight spin polarization, and the contribution from the $\mathrm{S}$ atom is more than that of the Ti atom. As given in Table $1, \mathrm{TiS}_{3}$ monolayer with a $\mathrm{Ti}$ vacancy exhibits a magnetic ground state and the calculated magnetic moment is $0.5 \mu_{\mathrm{B}} /$ supercell.

C. Double S Vacancy. Possible two-atom-vacancy structures of $\mathrm{TiS}_{3}$ are also investigated. First, the structure of double $\mathrm{S}$ atoms removed from the surface of $\mathrm{TiS}_{3}$ is studied. Figure $3 \mathrm{c}$ presents its fully relaxed configuration. Compared to the one $S$ vacancy situation, the lattice is more deformed. The double vacancy of surface $S$ atoms leads to a reconstruction of the bonds between the $S$ atoms of neighboring prisms (dark green one) and the $\mathrm{Ti}$ atoms closest to the vacancies. These $\mathrm{Ti}$ atoms become more strongly bonded to the surface $S$ atoms. However, compared to the defect-free structure, when two $S$ atoms are removed the lattice parameters are slightly changed. Bader charge analysis shows that removing two surface $S$ atoms does not affect the charges of the remaining $\mathrm{Ti}$ atoms. However, 0.7 excess electrons of the removed two $S$ atoms are shared by neighbor $\mathrm{S}$ atoms. The formation energy of double $\mathrm{S}$ vacancy is $8.48 \mathrm{eV}$. Thus, removing two $\mathrm{S}$ atoms from the $\mathrm{TiS}_{3}$ surface is more probable than removing one $\mathrm{Ti}$ atom.

The calculated density of states of monolayer $\mathrm{TiS}_{3}$ with double $S$ vacancy is presented in Figure 4d. When the second $S$ atom is taken out from the surface, $\mathrm{TiS}_{3}$ exhibits metallic character. As seen from Figure $4 \mathrm{~d}$, the main contribution comes from the $\mathrm{Ti}$ atoms and $\mathrm{TiS}_{3}$ with double $\mathrm{S}$ vacancy has an asymmetric DOS at the Fermi level. This asymmetric DOS at the Fermi level leads to a magnetic ground state with the magnetic moment value of $0.8 \mu_{\mathrm{B}}$ /supercell.

D. TiS Vacancy. Lastly, we considered the defected structure of $\mathrm{TiS}_{3}$ with a TiS vacancy. Figure $3 \mathrm{~d}$ shows that in the presence of TiS vacancy the position of the closest surface $S$ atom to the removed $S$ atom is significantly changed. This atom follows the direction of the arrow and becomes located at the top of the inner S atom (light green) with charge 6.6 electrons. Other two surface $S$ atoms move toward the vacancies, and their charges reduce to 6.0 and 6.2 electrons. The lattice parameters reduce to the values $a_{1}=14.90 \AA$ and $a_{2}=10.13 \AA$. Compared to the other vacancy types, its formation energy value is the highest with the value $16.15 \mathrm{eV}$.

The DOS diagram of $\mathrm{TiS}_{3}$ with $\mathrm{TiS}$ vacancy is illustrated in Figure 4e. Like $\mathrm{Ti}$ and double $\mathrm{S}$ vacancies, this vacancy type also leads to a metallic character. But, unlike the double $S$ vacancy, the Fermi level consists of orbitals of $S$ atom. As given in Table 1, the magnetic moment of this case is $0.3 \mu_{\mathrm{B}} /$ supercell.

\section{OXIDATION OF PRISTINE AND DEFECTIVE TiS}

It is well-known that two-dimensional ultrathin structures such as graphene, $\mathrm{MoS}_{2}$, phosphorene, etc., are prone to oxidation. ${ }^{4-43}$ Therefore, the search for structural and environmental stability of $\mathrm{TiS}_{3}$ is of importance. After the investigation of possible defect types in monolayer $\mathrm{TiS}_{3}$, in this section we address the oxidation process and the role of vacancies in that process.

A. O Atom Adsorption on Pristine and Defective $\mathrm{TiS}_{3}$. During experiments in highly oxidative conditions, the presence of the oxygen atom is inevitable and atomic $\mathrm{O}$ can be considered as a powerful tool to functionalize the surface of $\mathrm{TiS}_{3}$. Thus, we start to investigate the oxidation with atomic $\mathrm{O}$ on pristine $\mathrm{TiS}_{3}$. As shown in Figure 5a, the optimized structures show that an $\mathrm{O}$ atom is adsorbed by an $\mathrm{S}$ atom on the pristine $\mathrm{TiS}_{3}$ surface. The distance between $\mathrm{O}$ and $\mathrm{S}$ atoms is about $1.49 \AA$. The presence of the $\mathrm{O}$ atom does not have any significant effect on the lattice structure of $\mathrm{TiS}_{3}$. O atom binds strongly to the $S$ atom whose bond has an ionic character. The amount of charge transfer between $\mathrm{O}$ and $\mathrm{S}$ atoms is about 1.3 electrons from $\mathrm{S}$ to the $\mathrm{O}$ atom. The binding energy of the $\mathrm{O}$ atom on $\mathrm{TiS}_{3}$ crystal is $-3.89 \mathrm{eV}$. When $\mathrm{O}$ atom binds to the $\mathrm{TiS}_{3}$ surface, it loses its magnetic moment and the whole system does not exhibit any net magnetic moment.

The vacancy calculations have shown that the most probable defect type of $\mathrm{TiS}_{3}$ is the $\mathrm{S}$ atom vacancy. Since the $\mathrm{O}$ atom readily binds to the pristine $\mathrm{TiS}_{3}$ surface, the effect of the $S$ vacancy on the adsorption of atomic $\mathrm{O}$ is in order. Figure $5 \mathrm{~b}$ shows that, instead of being the nearest $S$ atom to the vacancy, $\mathrm{O}$ atom prefers to bind to the other surface $\mathrm{S}$ atom from tilted- 

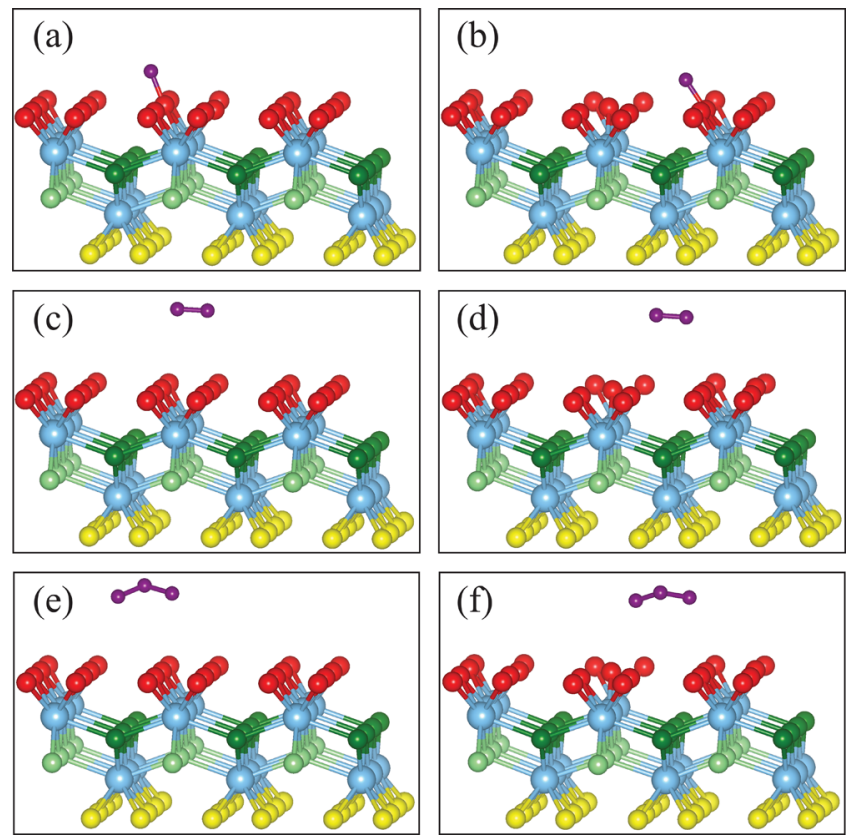

Figure 5. Tilted side view of (a) $\mathrm{O}$, (c) $\mathrm{O}_{2}$, and (e) $\mathrm{O}_{3}$ adsorbed on pristine $\mathrm{TiS}_{3}$. (b) $\mathrm{O}$, (d) $\mathrm{O}_{2}$, and (f) $\mathrm{O}_{3}$ adsorbed on an $\mathrm{S}$ vacant $\mathrm{TiS}_{3}$.

top site. Compared to the pristine case, the presence of the $S$ vacancy increases the binding energy of $\mathrm{O}$ atom from -3.89 to $-4.01 \mathrm{eV}$. The bond distance between $\mathrm{O}$ and $\mathrm{S}$ atoms is $1.48 \AA$. Unlike the pristine case, $\mathrm{O}$ atom distorts the lattice of defective $\mathrm{TiS}_{3}$, and the new lattice vectors are expanded to $a_{1}=14.93 \AA$ and $a_{2}=10.19 \AA$. $\mathrm{O}$ atom binds ionically to the surface $\mathrm{S}$ atom, and 1.1 electrons transfer from surface $S$ atom to the $O$ atom. Like in the pristine case, the net magnetic moment of the whole system is zero.

We also investigate substitutional adsorption of $\mathrm{O}$ atom on the surface of single layer $\mathrm{TiS}_{3}$. The formation energy of the substitutional $\mathrm{O}$ atom at $\mathrm{S}$ site is calculated using the formula $E_{\text {Subs }}=E_{\mathrm{M}+\mathrm{O}}-E_{\mathrm{M}}-E_{\mathrm{O}}+E_{\mathrm{S}}$, where $E_{\mathrm{M}+\mathrm{O}}$ denotes the energy of O-doped $\mathrm{TiS}_{3}, E_{\mathrm{M}}$ is the energy of pristine $\mathrm{TiS}_{3}$, and $E_{\mathrm{O}}$ and $E_{\mathrm{S}}$ are single atom energies. The substitution energy of $\mathrm{O}$ with $\mathrm{S}$ is found to be $-1.38 \mathrm{eV}$. The negative value for $\mathrm{O}$ atom indicates the spontaneous formation of substitutional doping at the $S$ site for $\mathrm{O}$ atom. It is also seen that the presence of substitutional $\mathrm{O}$ does not change the nonmagnetic structure of $\mathrm{TiS}_{3}$.

B. $\mathrm{O}_{2}$ Molecule Adsorption on Pristine and Defective $\mathrm{TiS}_{3}$. Approximately $21 \%$ of the earth's atmosphere is composed of $\mathrm{O}_{2}$ molecules. Thus, the binding mechanism of $\mathrm{O}_{2}$ molecule on $\mathrm{TiS}_{3}$ surface and the stability of $\mathrm{TiS}_{3}$ in the presence of this molecule are very crucial. The results of our calculations show that, compared to atomic $\mathrm{O}$, the $\mathrm{O}_{2}$ molecule binds very weakly to the $\mathrm{TiS}_{3}$ surface, and its binding energy is $-0.07 \mathrm{eV}$. Figure $5 \mathrm{c}$ shows that $\mathrm{O}_{2}$ locates $3.2 \AA$ above the $\mathrm{TiS}_{3}$ surface, and it prefers to bind on top of the vicinity of $S$ atom. The presence of $\mathrm{O}_{2}$ on the $\mathrm{TiS}_{3}$ surface does not cause any distortion on the lattice structure. Contrary to the single $\mathrm{O}$ atom case, there is no significant charge transfer between the surface and the molecule. Total magnetic moment of the system is $2 \mu_{\mathrm{B}}$, which is equal to the magnetic moment of an isolated $\mathrm{O}_{2}$ molecule.

The presence of an $S$ vacancy does not have any significant effect on the adsorption of $\mathrm{O}_{2}$ molecule on the $\mathrm{TiS}_{3}$ crystal.
Similar to the pristine case, $\mathrm{O}_{2}$ molecule binds rather weakly to the $S$ vacancy with a binding energy of $-0.05 \mathrm{eV}$. Compared to the pristine case, $\mathrm{O}_{2}$ molecule is localized more close to the $\mathrm{TiS}_{3}$ surface at a $z$-distance of $2.89 \AA$. The presence of $\mathrm{O}_{2}$ molecule does not cause any notable distortion of the defected $\mathrm{TiS}_{3}$ crystal. Like in the pristine case, there is almost no charge transfer between $\mathrm{TiS}_{3}$ and the $\mathrm{O}_{2}$ molecule, and the net magnetic moment of $\mathrm{O}_{2}$ molecule, which is $2 \mu_{\mathrm{B}}$, does not change in the presence of the $S$ vacancy.

C. $\mathrm{O}_{3}$ Molecule Adsorption on Pristine and Defective $\mathrm{TiS}_{3}$. To complete the analysis of oxidation of $\mathrm{TiS}_{3}$, the binding mechanism of the $\mathrm{O}_{3}$ molecule on the $\mathrm{TiS}_{3}$ surface is investigated. It is found that, compared to $\mathrm{O}_{2}$ molecule, $\mathrm{O}_{3}$ molecule binds more strongly to the $\mathrm{TiS}_{3}$ surface. The binding energy of $\mathrm{O}_{3}$ molecule on $\mathrm{TiS}_{3}$ surface is $-0.21 \mathrm{eV}$. As seen from Figure 5e, the $\mathrm{O}_{3}$ molecule locates $3.15 \AA$ above the $\mathrm{TiS}_{3}$ surface with two edge $\mathrm{O}$ atoms being closer to the surface compared to the middle $\mathrm{O}$ atom. The stability of $\mathrm{TiS}_{3}$ is not affected by $\mathrm{O}_{3}$. When the $\mathrm{O}_{3}$ molecule is placed on the $\mathrm{TiS}_{3}$ surface, it receives an extra 0.2 electrons from the $\mathrm{TiS}_{3}$. The net magnetic moment of the $\mathrm{O}_{3}$ molecule adsorbed pristine $\mathrm{TiS}_{3}$ is zero.

Finally, the adsorption of $\mathrm{O}_{3}$ molecule on an $\mathrm{S}$ vacancy is investigated. As seen from Figure $5 \mathrm{f}, \mathrm{O}_{3}$ molecule locates approximately $2.95 \AA$ above the $\mathrm{TiS}_{3}$ surface. In the $S$ vacancy case the end $\mathrm{O}$ atoms are placed closer to the surface compared to the middle $\mathrm{O}$ atom. The presence of the $\mathrm{S}$ vacancy slightly affects the binding energy of $\mathrm{O}_{3}$ molecule which is equal to $-0.20 \mathrm{eV}$. The $\mathrm{O}_{3}$ molecule does not make any significant effect on the lattice structure of $\mathrm{TiS}_{3}$ with an $\mathrm{S}$ vacancy. The charge transfer from the surface to the molecule is about 0.2 electrons. The adsorbed $\mathrm{O}_{3}$ molecule on an $\mathrm{S}$ vacancy does not possess any net magnetic moment.

\section{CONCLUSION}

In this paper, a detailed analyses of structural, electronic, and magnetic properties of pristine, defective, and oxidized structures of monolayer $\mathrm{TiS}_{3}$ are presented by using firstprinciples calculations. In addition to the PBE version of GGA, the HSE06 form of hybrid functionals was also used to describe the exchange-correlation density functional. Electronic structure calculations using the HSE06 hybrid functional indicated that monolayer $\mathrm{TiS}_{3}$ is a direct band gap semiconductor with a band gap of $1.05 \mathrm{eV}$. Our calculations also revealed interesting bonding nature of the monolayer $\mathrm{TiS}_{3}$ crystal that has ionic character inside and covalent character for surface atoms. The negatively charged surface of the crystal may also find some interesting applications such as nanoscale lubricants and charged coatings.

Among various vacancy defects including $\mathrm{S}, \mathrm{Ti}, \mathrm{TiS}$, and double $S$ vacancies, the single $S$ vacancy has the lowest formation energy. While the $S$ vacancy leads to an opening in the band gap, other vacancies result in metallicity in single layer $\mathrm{TiS}_{3}$. Pristine and $\mathrm{S}$ vacancy defected $\mathrm{TiS}_{3}$ does not possess any net magnetic moment, whereas other considered vacancies are magnetic. Our DFT oxidation studies revealed that $\mathrm{TiS}_{3}$ readily oxidizes with atomic $\mathrm{O}$. Moreover, it is found that oxidation of $\mathrm{TiS}_{3}$ with $\mathrm{O}_{3}$ is most likely to occur, while oxidation with $\mathrm{O}_{2}$ is less favorable on pristine and $\mathrm{S}$ defected $\mathrm{TiS}_{3}$ surface. The $\mathrm{S}$ vacancy has a slightly negative effect on the adsorption of $\mathrm{O}_{2}$ and $\mathrm{O}_{3}$ molecules on the $\mathrm{TiS}_{3}$ surface; however, it has a favoring effect on the adsorption of atomic $\mathrm{O}$. 


\section{AUTHOR INFORMATION}

\section{Corresponding Authors}

*E-mail fadiliyikanat@iyte.edu.tr (F.I.).

*E-mail hasan.sahin@uantwerpen.be (H.S.).

\section{Notes}

The authors declare no competing financial interest.

\section{ACKNOWLEDGMENTS}

This work was supported by the Flemish Science Foundation (FWO-Vl) and the Methusalem foundation of the Flemish government. Computational resources were provided by TUBITAK ULAKBIM, High Performance and Grid Computing Center (TR-Grid e-Infrastructure), and HPC infrastructure of the University of Antwerp (CalcUA), a division of the Flemish Supercomputer Center (VSC), which is funded by the Hercules foundation. H.S. is supported by a FWO Pegasus Marie Curie Fellowship. F.I., H.S., and R.T.S. acknowledge the support from TUBITAK through project 114F397.

\section{REFERENCES}

(1) Novoselov, K. S.; Geim, A. K.; Morozov, S. V.; Jiang, D.; Zhang, Y.; Dubonos, S. V.; Grigorieva, I. V.; Firsov, A. A. Electric Field Effect in Atomically Thin Carbon Films. Science 2004, 306 (5696), 666-669.

(2) Geim, A. K.; Novoselov, K. S. The Rise of Graphene. Nat. Mater. 2007, 6 (3), 183-191.

(3) Wang, Q. H.; Zadeh, K. K.; Kis, A.; Coleman, J. N.; Strano, M. S. Electronics and Optoelectronics of Two-Dimensional Transition Metal Dichalcogenides. Nat. Nanotechnol. 2012, 7 (11), 699-712.

(4) Xu, M.; Liang, T.; Shi, M.; Chen, H. Graphene-Like TwoDimensional Materials. Chem. Rev. 2013, 113 (5), 3766-3798.

(5) Huang, X.; Zeng, Z.; Zhang, H. Metal Dichalcogenide Nanosheets: Preparation, Properties and Applications. Chem. Soc. Rev. 2013, 42 (5), 1934-1946.

(6) Wilson, J. A.; Yoffe, A. D. The Transition Metal Dichalcogenides Discussion and Interpretation of the Observed Optical, Electrical and Structural Properties. Adv. Phys. 1969, 18 (73), 193-335.

(7) Zhang, Q.; Li, G.; Rhodes, D.; Kiswandhi, A.; Besara, T.; Zeng, B.; Sun, J.; Siegrist, T.; Johannes, M. D.; Balicas, L. Superconductivity with Extremely Large Upper Critical Fields in $\mathrm{Nb}_{2} \mathrm{Pd}_{0.81} \mathrm{~S}_{5}$. Sci. Rep. 2013, 3, 1682

(8) Ang, R.; Miyata, Y.; Ieki, E.; Nakayama, K.; Sato, T.; Liu, Y.; Lu, W. J.; Sun, Y. P.; Takahashi, T. Superconductivity and BandwidthControlled Mott Metal-Insulator Transition in $1 \mathrm{~T}-\mathrm{TaS}_{2 x} \mathrm{Se}_{x}$. Phys. Rev. B 2013, 88 (11), 115145.

(9) Splendiani, A.; Sun, L.; Zhang, Y.; Li, T.; Kim, J.; Chim, C.-Y.; Galli, G.; Wang, F. Emerging Photoluminescence in Monolayer $\mathrm{MoS}_{2}$. Nano Lett. 2010, 10 (4), 1271-1275.

(10) Ataca, C.; Sahin, H.; Ciraci, S. Stable, Single-Layer $\mathrm{MX}_{2}$ Transition-Metal Oxides and Dichalcogenides in a Honeycomb-Like Structure. J. Phys. Chem. C 2012, 116 (16), 8983-8999.

(11) di Salvo, F. J.; Moncton, D. E.; Waszczak, J. V. Electronic Properties and Superlattice Formation in the Semimetal TiSe ${ }_{2}$. Phys. Rev. B 1976, 14 (10), 4321.

(12) Wang, H.; Yu, L.; Lee, Y.-H.; Shi, Y.; Hsu, A.; Chin, M. L.; Li, L.-J.; Dubey, M.; Kong, J.; Palacios, T. Integrated Circuits Based on Bilayer $\mathrm{MoS}_{2}$ Transistors. Nano Lett. 2012, 12 (9), 4674-4680.

(13) Nicolosi, V.; Chhowalla, M.; Kanatzidis, M. G.; Strano, M. S.; Coleman, J. N. Liquid Exfoliation of Layered Materials. Science 2013, 340 (6139), 1226419.

(14) Chhowalla, M.; Shin, H. S.; Eda, G.; Li, L.-J.; Loh, K. P.; Zhang, H. The Chemistry of Two-Dimensional Layered Transition Metal Dichalcogenide Nanosheets. Nat. Chem. 2013, 5 (4), 263-275.

(15) Iyikanat, F.; Sahin, H.; Senger, R. T.; Peeters, F. M. Ag and Au Atoms Intercalated in Bilayer Heterostructures of Transition Metal Dichalcogenides and Graphene. APL Mater. 2014, 2 (9), 092801.
(16) Mak, K. F.; Lee, C.; Hone, J.; Shan, J.; Heinz, T. F. Atomically Thin $\mathrm{MoS}_{2}$ : A New Direct-Gap Semiconductor. Phys. Rev. Lett. 2010, 105 (13), 136805.

(17) Horzum, S.; Cakir, D.; Suh, J.; Tongay, S.; Huang, Y.-S.; Ho, C.H.; Wu, J.; Sahin, H.; Peeters, F. M. Formation and Stability of Point Defects in Monolayer Rhenium Disulfide. Phys. Rev. B 2014, 89 (15), 155433.

(18) Zeng, H.; Dai, J.; Yao, W.; Xiao, D.; Cui, X. Valley Polarization in $\mathrm{MoS}_{2}$ Monolayers by Optical Pumping. Nat. Nanotechnol. 2012, 7 (8), 490-493.

(19) Brattas, L.; Kjekshus, A. On the Properties of Compounds with the $\mathrm{ZrSe}_{3}$ Type Structure. Acta Chem. Scand. 1972, 26 (9), 34413449.

(20) Furuseth, S.; Brattas, L.; Kjekshus, A. On the Crystal Structures of $\mathrm{TiS}_{3}, \mathrm{ZrS}_{3}, \mathrm{ZrSe}_{3}, \mathrm{ZrTe}_{3}, \mathrm{HfS}_{3}$, and $\mathrm{HfSe}_{3}$. Acta Chem. Scand., Ser. A 1975, 29, 623-631.

(21) Finkman, E.; Fisher, B. Electrical Transport Measurements in $\mathrm{TiS}_{3}$. Solid State Commun. 1984, 50 (1), 25-28.

(22) Gorlova, I. G.; Zybtsev, S. G.; Pokrovskii, V. Y.; Bolotina, N. B.; Verin, I. A.; Titov, A. N. Nonlinear Conductivity of Quasi-OneDimensional Layered Compound $\mathrm{TiS}_{3}$. Physica B 2012, 407 (11), $1707-1710$

(23) Gorlova, I. G.; Pokrovskii, V. Y.; Zybtsev, S. G.; Titov, A. N.; Timofeev, V. N. Features of the Conductivity of the Quasi-OneDimensional Compound $\mathrm{TiS}_{3}$. J. Exp. Theor. Phys. 2010, 111 (2), 298-303.

(24) Ferrer, I. J.; Ares, J. R.; Clamagirand, J. M.; Barawi, M.; Sanchez, C. Optical Properties of Titanium Trisulphide $\left(\mathrm{TiS}_{3}\right)$ Thin Films. Thin Solid Films 2012, 535, 398-401.

(25) Ferrer, I. J.; Marciá, M. D.; Carcelén, V.; Ares, J. R.; Sánchez, C. On the Photoelectrochemical Properties of $\mathrm{TiS}_{3}$ Films. Energy Procedia 2012, 22, 48-52.

(26) Guilmeau, E.; Berthebaud, D.; Misse, P. R. N.; Hébert, S.; Lebedev, O. I.; Chateigner, D.; Martin, C.; Maignan, A. $\mathrm{ZrSe}_{3}$-Type Variant of $\mathrm{TiS}_{3}$ : Structure and Thermoelectric Properties. Chem. Mater. 2014, 26 (19), 5585-5591.

(27) Island, J. O.; Buscema, M.; Barawi, M.; Clamagirand, J. M.; Ares, J. R.; Sánchez, C.; Ferrer, I. J.; Steele, G. A.; van der Zant, H. S. J.; Gomez, A. C. Ultrahigh Photoresponse of Few-Layer $\mathrm{TiS}_{3}$ Nanoribbon Transistors. Adv. Opt. Mater. 2014, 2 (7), 641-645.

(28) Island, J. O.; Barawi, M.; Biele, R.; Almazan, A.; Clamagirand, J. M.; Ares, J. R.; Sanchez, C.; van der Zant, H. S. J.; Alvarez, J. V.; D'Agosta, R.; et al. $\mathrm{TiS}_{3}$ Transistors with Tailored Morphology and Electrical Properties. Adv. Mater. 2015, 27 (16), 2595-2601.

(29) Barawi, M.; Flores, E.; Ferrer, I. J.; Ares, J. R.; Sanchez, C. Titanium Trisulphide $\left(\mathrm{TiS}_{3}\right)$ Nanoribbons for Easy Hydrogen Photogeneration under Visible Light. J. Mater. Chem. A 2015, 3 (15), 7959-7965.

(30) Gorlava, I. G.; Zybtsev, S. G.; Pokrovskii, V. YA. Conductance Anisotropy and the Power-Law Current-Voltage Characteristics along and across the Layers of the $\mathrm{TiS}_{3}$ Quasi-One-Dimensional Layered Semiconductor. JETP Lett. 2014, 100 (4), 256-261.

(31) Gorlava, I. G.; Zybtsev, S. G.; Pokrovskii, V. YA.; Bolotina, N. B.; Gavrilkin, S. Yu.; Tsvetkov, A. Yu. Magnetotransport and PowerLaw I-V Curves of the Layered Quasi One-Dimensional Compound $\mathrm{TiS}_{3}$. Physica B 2015, 460, 11-15.

(32) Tanibata, N.; Matsuyama, T.; Hayashi, A.; Tatsumisago, M. AllSolid-State Sodium Batteries Using Amorphous $\mathrm{TiS}_{3}$ Electrode with High Capacity. J. Power Sources 2015, 275, 284-287.

(33) Wu, J.; Wang, D.; Liu, H.; Lau, W.-M.; Liu, L.-M. An Ab Initio Study of $\mathrm{TiS}_{3}$ : a Promising Electrode Material for Rechargeable Li and $\mathrm{Na}$ Ion Batteries. RSC Adv. 2015, 5 (28), 21455-21463.

(34) Jin, Y.; Li, X.; Yang, J. Single Layer of $\mathrm{MX}_{3}(\mathrm{M}=\mathrm{Ti}, \mathrm{Zr}$; $\mathrm{X}=\mathrm{S}$, Se, Te): a New Platform for Nano-Electronics and Optics2015, arXiv:1502.05108 [cond-mat.mtrl-sci]. arXiv.org e-Print archive. http://arxiv.org/abs/1502.05108 (accessed Feb 18, 2015).

(35) Kresse, G.; Furtmuller, J. Efficient Iterative Schemes for $\mathrm{Ab}$ Initio Total-Energy Calculations Using a Plane-Wave Basis Set. Phys. Rev. B 1996, 54 (16), 11169. 
(36) Kresse, G.; Joubert, D. From Ultrasoft Pseudopotentials to the Projector Augmented-Wave Method. Phys. Rev. B 1999, 59 (3), 1758. (37) Perdew, J. P.; Burke, K.; Ernzerhof, M. Generalized Gradient Approximation Made Simple. Phys. Rev. Lett. 1996, 77 (18), 3865.

(38) Heyd, J.; Scuseria, G. E.; Ernzerhof, M. Erratum: "Hybrid Functionals Based on a Screened Coulomb Potential”. J. Chem. Phys. 2006, 124 (21), 219906.

(39) Henkelman, G.; Arnaldsson, A.; Jonsson, H. A Fast and Robust Algorithm for Bader Decomposition of Charge Density. Comput. Mater. Sci. 2006, 36 (3), 354-360.

(40) Grimme, S. Semiempirical GGA-Type Density Functional Constructed with a Long-Range Dispersion Correction. J. Comput. Chem. 2006, 27 (15), 1787-1799.

(41) Vinogradov, N. A.; Schulte, K.; Ng, M. L.; Mikkelsen, A.; Lundgreen, E.; Martensson, N.; Preobrajenski, A. B. Impact of Atomic Oxygen on the Structure of Graphene Formed on $\operatorname{Ir}(111)$ and Pt(111). J. Phys. Chem. C 2011, 115 (19), 9568-9577.

(42) Wang, G.; Pandey, R.; Karna, S. P. Effects of Extrinsic Point Defects in Phosphorene: B, C, N, O and F Adatoms 2015, arXiv:1503.04165 [physics.chem-ph]. arXiv.org e-Print archivehttp:// arxiv.org/abs/1503.04165 (accessed Mar 13, 2015).

(43) Yamamoto, M.; Einstein, T. L.; Fuhrer, M. S.; Cullen, W. G. Anisotropic Etching of Atomically Thin $\mathrm{MoS}_{2}$. J. Phys. Chem. C 2013, 117 (48), 25643-25649. 